\title{
Molecular Dynamics Studies on Fundamental Molecular Surface Processes
}

\author{
M. Cacciatore and M. Rutigliano ${ }^{\mathrm{a}}$ \\ ${ }^{a}$ CNR-IMIP Institute of Inorganic Methodologies and Plasmas, Department of Chemistry, \\ University of Bari, via Orabona N.6, 70126 Bari, Italy \\ mario.cacciatore@ba.imip.cnr.it
}

\begin{abstract}
In this contribution, we give examples of results recently obtained in fundamental molecular dynamics studies and DFT electronic structure calculations performed on prototype surface processes due to the interaction of atomic and molecular oxygen, nitrogen, hydrogen, $\mathrm{C}$ on substrates of different nature. The discussed systems are known to be among the most important heterogeneous systems relevant to laboratory and natural gas-surface systems. The surface processes followed in the MD simulations include atom recombination reactions, dissociative chemisorption, adsorption and adsorption/desorption of atoms and molecules. Correlations between the dynamics of the surface processes and the molecular properties of the gas-phase particles and the structural behaviours of the surfaces will be highlighted.
\end{abstract}

Keywords: molecule-surface interactions, molecular dynamics, atom recombination

PACS: 82.65.+2; 34.35.+a; 82.20.Bc

\section{GENERAL CONSIDERATIONS}

The chemi and physisorption of atoms and molecules on solid surfaces can lead to a large number of chemicophysical processes of great interest in many important research fields both from a fundamental and technological point of view: material science and plasma-chemical processes, aerothermodynamics, interstellar chemistry and others. Among the many possible molecular processes active at surfaces, we mention the direct and indirect inelastic scattering (desorption and adsorption/desorption processes), sticking, chemical reactions, charge transfer and charge neutralization, formation of volatile surface radicals. Each of these processes is active in a specific range of collisional energies according to the behaviours of the surfaces involved in the interaction. The data needed in kinetic modeling of the chemistry at the gas-surface interlayer, as for example in kinetic Monte Carlo simulation, are: state-selected and global recombination coefficients, state-to-state dissociation probabilities, distributions in angles and energies of the scattered particles, energy accommodation coefficient, diffusion coefficients etc. Of key importance is the dependence of the collisional coefficients on the surface parameters ( surface site and surface structure, corrugation, surface contamination), and on the molecular parameters of the gas-phase particles hitting the surface (translational and internal energy content, incident angles). Unfortunately, contrary to gas-phase processes where large database of state-to-state rate constants and cross-sections are available[see for example [1,2], the collisional data for heterogeneous processes needed by the plasma-chemistry and plasma-physics community are rather sparse.

In this contribution we focus on two classes of heterogeneous processes: inelastic direct desorption and indirect adsorption-desorption, and molecule formation after atom recombination at surfaces. Special attention is given to the latter reactive process. This reaction can be both an important channel for surface atom abstraction and an effective source of energetically activated molecules. Recombination reactions can follow three basic mechanisms.

According to the Langmuir-Hinshelwood (L-H) mechanism the reaction takes place between two pre-adsorbed atoms $\left(\mathrm{O}_{\mathrm{ad}}\right.$. oxygen is assumed here as a reference chemical species ) which diffuse on the surface from site to site before recombining.

$$
\mathrm{O}_{\mathrm{ad}} \text { *wall }+\mathrm{O}_{\mathrm{ad}}{ }^{*} \text { wall -----> } \mathrm{O}_{2}(\mathrm{v}, \mathrm{j})+\text { wall }+\Delta \mathrm{E}
$$


The Eley-Rideal (E-R) reaction is a two-step mechanism: first an atom is adsorbed at the surface in a specific "active" surface site, then an atom approaching the surface from the gas-phase $\left(\mathrm{O}_{\mathrm{gas}}\right)$ reacts with the pre-adsorbed atom thus forming a molecule in a specific roto-vibrational state $(\mathrm{v}, \mathrm{j})$.

$$
\mathrm{O}_{\mathrm{gas}}+\mathrm{O}_{\mathrm{ad}}{ }^{*} \text { wall -----> } \mathrm{O}_{2}(\mathrm{v}, \mathrm{j})+\text { wall }+\Delta \mathrm{E}^{\prime}
$$

The E-R reaction can be direct or indirect according to the different dynamical pathways followed in the interaction. According to the latter mechanism, the gas atom hits the surface several times, acquires enough kinetic energy to diffuse on the surface towards the previously chemisorbed atom with which it finally recombines. The indirect, precursor-mediate mechanism [3,4], is also known as Hot Atom (HA) recombination mechanism

$$
\mathrm{O}_{\mathrm{gas}}+\mathrm{O}_{\mathrm{ad}}{ }^{*} \text { wall -----> }[\mathrm{O}]_{\mathrm{ad}}+\mathrm{O}_{\mathrm{ad}}{ }^{*} \text { wall -----> } \mathrm{O}_{2}(\mathrm{v}, \mathrm{j})+\Delta \mathrm{E},
$$

Recombination reactions can be strongly exothermic, the upper limit of the energy released in the reaction being given by the energy of the chemical bond formed in the recombination. $\Delta \mathrm{E}$ is given by $\Delta \mathrm{E}=\mathrm{D}_{0}-\mathrm{E}_{\mathrm{b}}+\mathrm{E}_{\mathrm{kin}}$, where $\mathrm{D}_{0}$ is the bond energy of the isolated molecule, $\mathrm{E}_{\mathrm{b}}$ is the binding energy of the chemisorbed or physisorbed atom, $\mathrm{E}_{\mathrm{kin}}$ is the impact energy of the incoming gas atom. It is worth noting that $\Delta \mathrm{E}$ cannot be easily defined since the energy released in the recombination process depends upon different factors, among the others the number of active sites, the site where the atom is adsorbed, and the nature of the atomic adsorption, that is whether the atom is chemi or physisorbed. A further factor that makes the energetic of surface processes quite complex concerns the energy sharing mechanism among the various degrees of freedom involved in the dynamics. $\Delta \mathrm{E}$ can be shared between the internal and translational states of the product molecules and the degrees of freedom of the surface. Thus, a fraction of $\Delta \mathrm{E}$ can be transferred to the surface due to the phonons and/or electron-holes excitation/de-excitation processes, the other part is gained by the vibrational, rotational and the translational motions of the newly formed molecules.

$$
\Delta \mathrm{E}---->\left[\begin{array}{l}
\text { Internal states of the scattered particles: } \\
\text { vibration, rotation, translation, electronic excitation } \\
\text { Heat flux to the surface: phonons and/or electron-holes excitation }
\end{array}\right.
$$

Therefore, the evaluation of $\Delta \mathrm{E}$ requires a fully dimension dynamical simulation of the surface reaction which includes the phonons and/or electrons and the six variables of the gas-phase atoms. It must be also remarked that a fully quantum mechanics approach to the dynamics of heterogeneous systems is yet quite difficult, so that quantum calculations are performed, very often, assuming rigid surfaces and no energy exchange between the chemical particles and the surface. With the semiclassical approach [5] the inclusion in the collision dynamics of such energy exchanges can be considered. The determination of the energy flow pathways is of primary importance for understanding the chemistry of various heterogeneous systems, in particular for the understanding of the heat flux to the tiles ( silica-based) of the thermal protection system of shuttles under re-entry space missions.

\section{SEMICLASSICAL MOLECULAR DYNAMICS CALCULATIONS}

A realistic description of elementary surface processes, in particular of the multi-phonons and electron-hole pairs excitation-deexcitation processes, can be achieved in the framework of a semiclassical collisional model $[5,6]$ according to which the degrees of freedom of the gas-phase particles colliding with the surface are assumed classical, whereas the dynamics of the lattice atoms and of the electron-holes is treated quantum-mechanically. The classical and quantum systems are coupled by solving self-consistently the Hamilton's equations of motion and the time-dependent quantum equations. This coupling is possible by the definition of an effective Hamiltonian, of the mean-field type, which, for a two-atoms/surface system, is given by:

$$
H_{e f f}=\sum_{i=1,2} \frac{1}{2 m_{i}}\left(p_{x_{i}}^{2}+p_{y_{i}}^{2}+p_{z_{i}}^{2}\right)+V_{a d d}^{p h}+V_{a d d}^{e-h}+E_{\mathrm{int}}
$$


where $\mathrm{R}_{\mathrm{i} \alpha}$ is the distance between the gas-phase atom $i$ and the lattice atom $\alpha, \quad\left(\mathrm{x}_{\mathrm{i}}, \mathrm{y}_{\mathrm{i}}, \mathrm{z}_{\mathrm{i}}\right)$ are the Cartesian coordinates of the gas-phase atom, $r$ is the atom-atom distance in the gas-phase. $\mathrm{E}_{\mathrm{int}}$ is the energy exchanged with the surface phonons and electron-hole pairs, $V_{a d d}^{e-h}$ and $V_{a d d}^{p h}\left(t, T_{S}\right)$ are the "dynamical effective" potential due to the electron-hole pairs and phonons excitation/de-excitation processes, respectively. $V_{a d d}^{p h}\left(t, T_{S}\right)$ is given as the expectation value of the gas-surface interaction potential, $\mathrm{V}_{\text {pot }}$, over the total wave function of the phonon state given as a statistical distribution of pure states at a given temperature Ts. $V_{a d d}^{p h}\left(t, T_{S}\right)$ is given as:

$$
V_{a d d}^{p h}\left(t, T_{S}\right)=V_{0}\left(R_{i, \alpha}, r\right)+\sum_{k} V_{k}^{(1)} \eta_{k}\left(t, T_{S}\right)
$$

$\eta_{\mathrm{k}}$ are the "phonon excitation strengths" given in terms of the Fourier components of the external forces exerted between the phonons and the chemical particles, $V_{o}$ is the interaction potential with the lattice atoms in their equilibrium positions, $V_{k}^{(1)}$ is the first derivative of the interaction potential with respect to phonon mode $k$.

Therefore, since $\mathrm{V}_{\text {add }}$ depends upon the interaction time $\mathrm{t}$ and $\mathrm{Ts}$, with this method it is possible to explore the intrinsic effect of the surface temperature on the surface catalytic processes.

In the simulation the scattering equations are solved under specific, well defined initial conditions of the gasphase and chemisorbed atoms: position coordinates of the gas-phase atoms taken randomly within the surface unit cell, incident angles, translational and internal energies of the particles hitting the surface, lattice site where the interaction takes place, surface temperature, coverage.

\section{(Some) CASES STUDIED}

The interaction of $\mathrm{O}$ atoms with silica surfaces, $\beta$-cristobalite and $\beta$-quartz, at surface temperatures typically met in re-entry aerospace missions has been extensively studied both from the experimental and theoretical point of view [7, and reference therein]. A prerequisite in molecular dynamics studies [8,9] is the determination of the interaction potential. A first attempt to characterize such interactions using basic physical assumptions was made in Ref.[7] and Ref.[10]. In these studies, a semiempirical potential for $\mathrm{O}, \mathrm{O}_{2}$ interacting with two different silica polymorphs, $\beta$ cristobalite and $\beta$-quartz, was derived and used in the semiclassical scattering code to simulate the E-R and L-H recombination reaction of oxygen adsorbed within the unit cell of the two silica polymorphs.

Two silica model surfaces, large enough for studying collisional processes, were built up from the silica unit cell of the two polymorphs. Then, the phonon eigenvalues and eigenvectors needed in the scattering equations were calculated by diagonalizing the dynamical force-constant matrix obtained from the potential of the two polymorphs

The global recombination coefficient $\gamma$ was calculated at various surface temperatures, Ts $=600,800,1000,1600 \mathrm{~K}$, assuming both the E-R and the L-H mechanism. Comparison between the theoretical $\gamma$ values and the experimental results shows that at the higher Ts the recombination reaction should follow the E-R mechanism, whereas at $\mathrm{Ts}=600,800 \mathrm{~K}$ a good match was found between the $\mathrm{L}-\mathrm{H} \gamma$ coefficient and the experimental ones. It is worth noting that the direct comparison between results obtained in MD calculations and the $\gamma$ coefficients measured in cell experiments must be taken with some precaution, due to the fact that the surface target used in the experiments is, very likely, a poly-crystalline, corrugated surface, whereas in MD calculations the silica surface sample is a perfect, crystalline surface. The different atom arrangement in the surface, and therefore the different flexibility of the electronic band structure, has an impact on the surface catalytic activity. Thus, $\beta$-quartz has a weaker catalytic activity towards $\mathrm{O}$ atom recombination than $\beta$-cristobalite. This is shown in TABLE 1 where the experimental and semiclassical $\gamma$ coefficient is reported at $\mathrm{Ts}=1000 \mathrm{~K}$. From the reported data [7] we note the ability of the semiclassical method to account for the different catalytic behaviour of the two silica polymorphs.

TABLE 1. Recombination coefficient $\gamma$ for $O$ atom recombination on $\beta$-cristobalite and $\beta$-quartz at Ts=1000K:semiclassical $\left(\gamma_{\mathrm{th}}\right)$ and experimental data $[7,10]$

\begin{tabular}{lcc}
\hline Surface & $\gamma_{\exp }$ & $\gamma_{\text {th }}$ \\
\hline$\beta$-quartz & 0.007 & 0.008 \\
$\beta$-cristobalite & & \\
\hline
\end{tabular}


A part from the good agreement reached between simulation and experiment, the probably most interesting result obtained in this study was the first calculation of the energy accommodation coefficient $\beta$, a key factor for the thermal protection system (TPS) of shuttles used in space missions.

$\beta$ is defined as the fraction of the total energy delivered in the various $\mathrm{O}, \mathrm{O}_{2}$-silica processes which is transferred as heat flux to the surface. This energy fraction can be the cause of the thermal damage of the silica substrate. Determining $\beta$ is not an easy task, both from the experimental and theoretical point of view, so that the energy accommodation coefficient is generally not well known. As a consequence, the evaluation of the heat flux to the silica surface is made under two extreme empirical approximations for the $\beta$ coefficient: complete energy accommodation $(\beta=1)$ and un-complete accommodation $(\beta=0)$. In Ref. $[7,10] \beta$ was found $<<1$, about 0.3 .

Although the collisional dynamical model develop in these studies was in reasonable agreement with the experimental findings, the interaction potential was a rather crude approximation of the real interaction forces.

We have therefore focused our investigation on $a b$ initio electronic structure calculations based on different DFT functionals for the two systems: $\mathrm{O}, \mathrm{O}_{2}-\beta$ cristobalite/ $\beta$-quartz and $\mathrm{N}_{2} \mathrm{~N}_{2}-\beta$ cristobalite. The calculations were performed within the size-scaled cluster approach $[11,12]$. Spin polarized wave functions were used as basis sets.
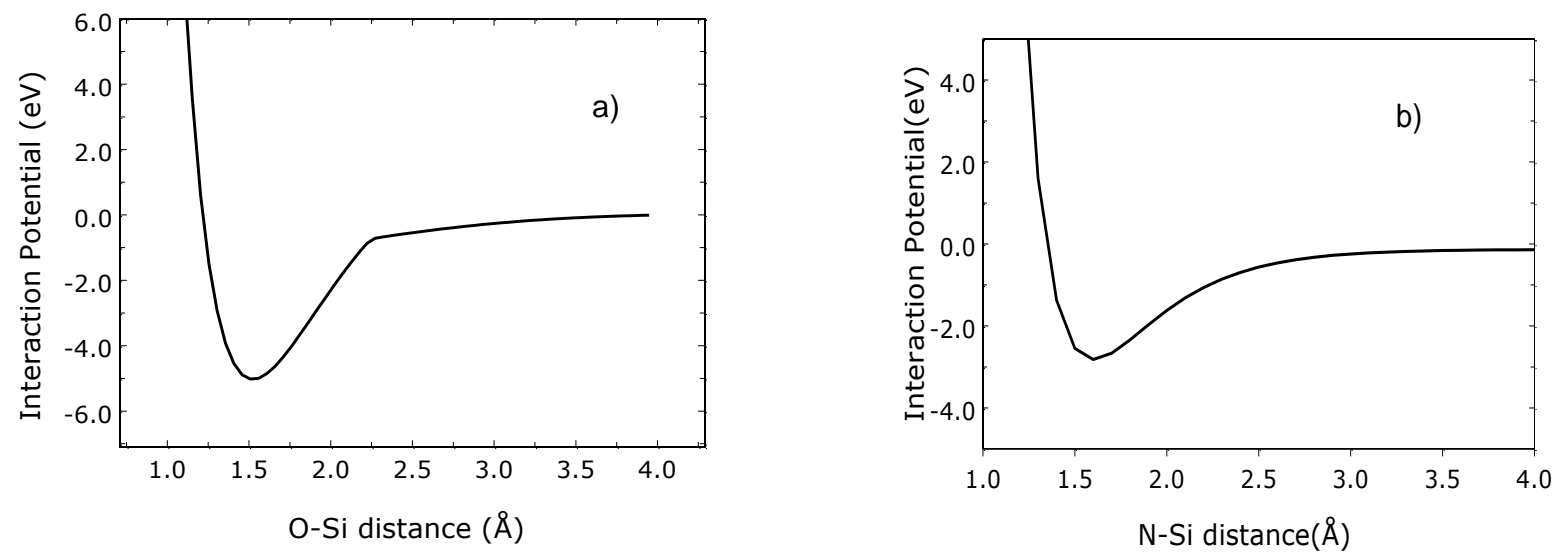

FIGURE 1. Left panel a) : DFT- PBE0/6-311+G* interaction potential of $\mathrm{O}_{\text {gas }}$ interacting perpendicularly with a Si atom of $\beta$-cristobalite as a function of the $\mathrm{O}_{\mathrm{gas}}-\mathrm{Si}$ distance. Right panel b): same as a) but for $\mathrm{N}_{\mathrm{gas}}-\mathrm{Si}$ interaction.

In FIGURE 1a,b) the interaction potential of $\mathrm{O}$ and $\mathrm{N}$ approaching perpendicularly a $\mathrm{Si}$ surface atom of the $\beta$ cristobalite is drown. We notice that atomic oxygen and atomic nitrogen are chemisorbed on top of a Si active site with a binding energy close to $6.0 \mathrm{eV}$ and $2.7 \mathrm{eV}$, respectively. On the other hand, molecular oxygen can be slightly chemisorbed on condition that an activation energy of about $2 \mathrm{eV}$ is gained by the molecule to surmount the energy barrier [11]. These results have some important consequences on the dynamics of the surface processes. In particular we note that the adsorption process of atomic $\mathrm{O}$ is a strongly exothermic process, whereas the recombination reaction with $\mathrm{O}$ chemisorbed on top of a $\mathrm{Si}$ surface atom can be endothermic. In FIGURE 2. a typical collisional event is shown where a gas-phase oxygen atom approaches the silica surface perpendicularly over a Si lattice site and hits the surface several times before being definitively adsorbed. The energy transferred to the surface phonons, calculated at each time step of the O-silica interaction, is also reported in the figure. 


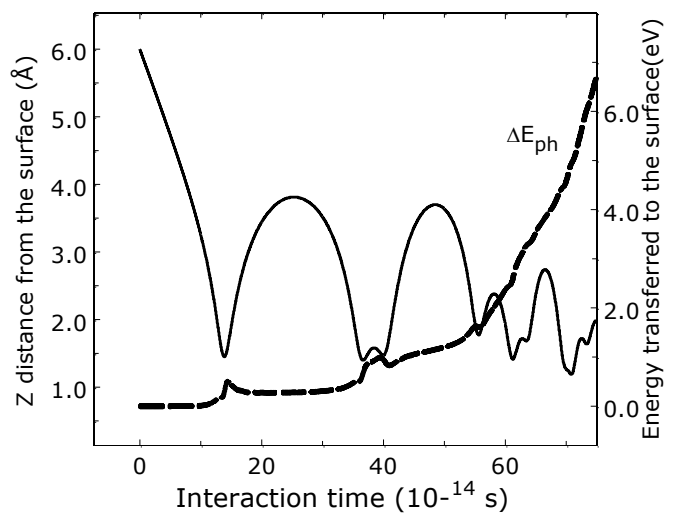

FIGURE 2. Time evolution of a typical multi-step adsorption process: $\mathrm{O}_{\mathrm{gas}}+$ silica -----> $\mathrm{O}_{\mathrm{ad}}{ }^{*}$ silica $+\Delta \mathrm{E}_{\mathrm{ph}}$. The $\mathrm{Z}$ distance from the surface of the $\mathrm{O}$ gas-atom colliding with the silica surface is reported as a function of the collision time (left-hand scale). The energy transferred to the surface (dotted line) is also reported on the right-hand scale. $\mathrm{E}_{\mathrm{kin}}=0.5 \mathrm{eV}$, $\mathrm{T}_{\mathrm{S}}=1000 \mathrm{~K}$.

We note that, unlike the recombination reaction that could be endothermic, the adsorption process is strongly exothermic with a large fraction of the exothermic energy transferred to the silica substrate. MD simulations performed by us show that the adsorption probability of $\mathrm{O}$ over $\beta$-cristobalite is quite high. At Ts=1000K, $\mathrm{E}_{\mathrm{kin}}=0.2 \mathrm{eV}$ and $\mathrm{O}$ approaching perpendicularly a $\mathrm{Si}$ lattice site, the adsorption probability is close to 0.9 . The corresponding $\mathrm{O}$ adsorption probability over a quartz surface is much smaller, close to 0.6.

It is clear that these new results open new questions and perspectives in the current discussion regarding the "realistic" estimation of the heat transferred to the silica substrate. In fact, many competing surface processes, and here specifically $\mathrm{O}$ adsorption, other than $\mathrm{O}$ recombination reaction can contribute to the total heat balance at the silica-air boundary layer. Further results obtained in our work and by Sayòs and co-workers [13] show that the Osilica interaction is rather complex in that the binding energy of the adsorbed $\mathrm{O}$ atom depends, very much, on the site ( whether $\mathrm{Si}$ or $\mathrm{O}$ lattice site) where the oxygen is adsorbed and on the angle with which the $\mathrm{O}$ atom approaches a specific adsorption site. As a consequence, the evaluation of the total energy transferred to the surface requires a fully dimension, multi-processes approach to the $\mathrm{O}, \mathrm{O}_{2}$-silica interaction.

An important aspect of catalytic reactions is the surface temperature effect. This effect is quite complex and remains quite difficult to describe in molecular dynamics simulations. In fact, this effect can be due to various micro and macro factors: reaction mechanism and adsorbate-substrate energy exchange mechanism. surface coverage and mobility of the adsorbed species, surface structural modifications,

The energy exchange process between adsorbate and substrate, in particular the interaction with the surface phonons, controls the surface temperature effect. The adsorbate-phonons coupling is very effective when the Debye frequency of the solid is close to the stretching frequency of the adsorbate-substrate vibrational mode. Nevertheless, a weak phonon coupling can have an impact on the surface temperature effect and on the isotopic effect on surface processes. This has been found for $\mathrm{H}_{2}$ formation after $\mathrm{H}$ atom recombination on graphite. The hydrogen/graphite system is characterized by a large mismatch between the Debye phonon frequency $(\sim 55 \mathrm{THz})$ and the H-graphite stretching frequency $(\sim 790 \mathrm{THz}$, which corresponds to the $\mathrm{H}$ chemisorption energy). On the other hand, the graphite substrate is characterized by a soft phonon structure.

In FIGURE 3 the semiclassical recombination probability for $\mathrm{H}_{2}$ formation over a graphite surface is reported at two surface temperatures, $\mathrm{Ts}=100 \mathrm{~K}$ and $\mathrm{Ts}=800 \mathrm{~K}$. Although the masses involved in the interaction are very light, the surface temperature has a large impact on the recombination dynamics. In fact, the energy threshold of the process is shifted toward higher energies and the maximum of the recombination probability is lowered by a factor close to 2 as Ts increases from $100 \mathrm{~K}$ to $800 \mathrm{~K}$. 


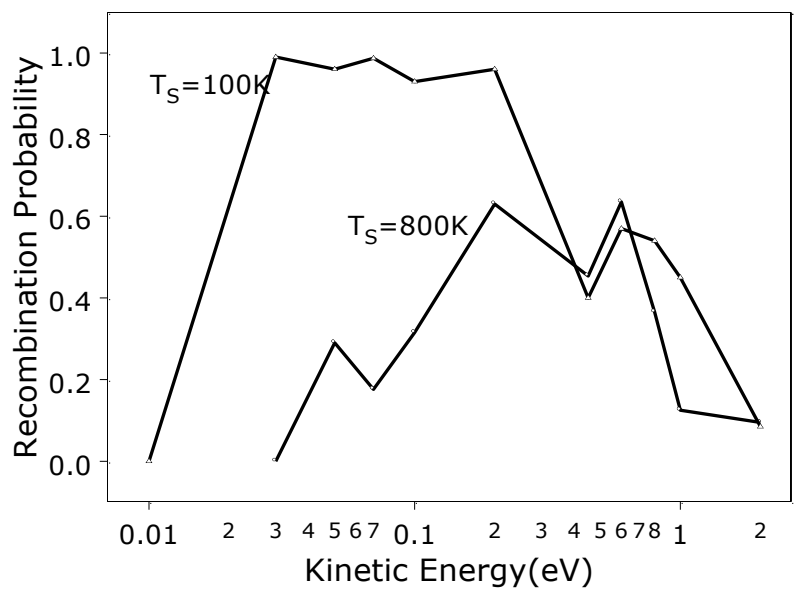

FIGURE 3. E-R recombination probability for $\mathrm{H}_{2}$ formation on a graphite (0001) surface at $\mathrm{Ts}=100,800 \mathrm{~K}$ reported as a function of the kinetic energy of the gas-phase $\mathrm{H}$ atom

This reaction is of key importance in the chemistry of the interstellar media (ISM) and has been the object of intense investigation and discussion [14,15]. An important aspect concerns the roto-vibrational distribution in the final states of the formed $\mathrm{H}_{2}$ molecules. This effect has been investigated in great detail in several studies [16,17] and by us [18]. The vibrational population distribution of $\mathrm{H}_{2}$ formed on graphite (0001) reported in FIGURE 4 shows that $\mathrm{H}_{2}$ can be formed in highly vibrationally excited states.
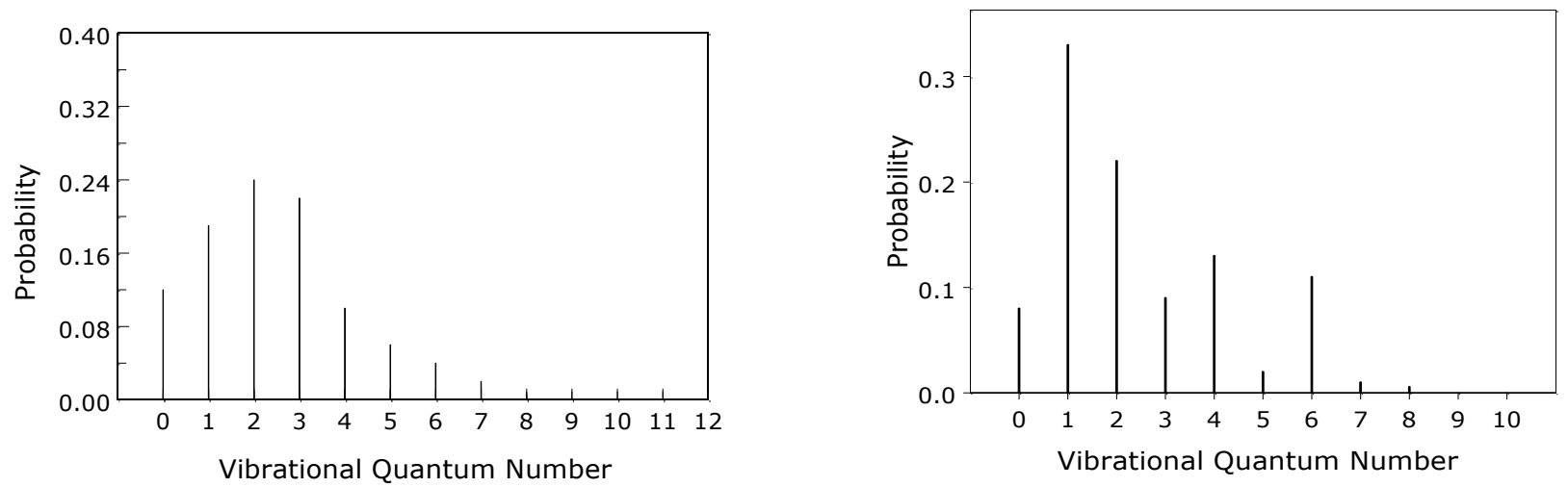

FIGURE 4. Vibrational distribution of $\mathrm{H}_{2}$ formed in the reaction: $\mathrm{H}_{\mathrm{gas}}+\mathrm{H}_{\mathrm{ad}} *$ graphite -----> $\mathrm{H}_{2}(\mathrm{v}, \mathrm{j})+$ graphite. Left panel: $\mathrm{T}_{\mathrm{S}}=100 \mathrm{~K}, \mathrm{E}_{\mathrm{kin}}=70 \mathrm{meV}$. Right panel: $\mathrm{T}_{\mathrm{S}}=10 \mathrm{~K}, \mathrm{E}_{\text {kin }}=70 \mathrm{meV}$

This result can be relevant for the understanding the IR emission of the interstellar clouds. Nevertheless, we have to remark that in the simulation $\mathrm{H}$ was assumed chemisorbed on top of a $\mathrm{C}$-atom placed at the corner of the exagonal graphite ring $\left(\mathrm{E}_{\mathrm{b}} \approx 0.6 \mathrm{eV}\right)$. Since recent investigations seem to confirm the existence of an energy barrier to chemisorption of about $0.2 \mathrm{eV}$, the chemisorption process could not be allowed under ISM conditions. On the other hand, $\mathrm{H}$ can be very likely physisorbed on graphite $\left(\mathrm{E}_{\mathrm{b}} \approx 0.40 \mathrm{meV}\right)$, so that an additional route to the $\mathrm{H}_{2}$ formation in the ISM media can be recombination of physisorbed $\mathrm{H}$ atoms both via E-R and L-H mechanism $[19,20]$. From the experimental point of view, no firm conclusion of the energy partitioning in the $\mathrm{H}$ recombination reaction can be 
inferred, although laboratory investigations [14,15] and indirect spectroscopic observations in astronomy indicate that $\mathrm{H}_{2}$ should be formed in vibrational states less excited than predicted from theory.

The isotope effect on the surface reaction processes is one of the current issue in gas-surface interaction. This effect was explored by us [18b] for the H-graphite system by considering different isotope reactions. In FIGURE 5 the E-R probability for $\mathrm{D}_{2}$ and $\mathrm{H}_{2}$ formation is shown as a function of the collisional energy.

$$
\begin{gathered}
\mathrm{D}_{\text {gas }}+\mathrm{D}_{\mathrm{ad}}{ }^{*} \text { graphite -------> } \mathrm{D}_{2}(\mathrm{v}, \mathrm{j})+\text { graphite } \\
\mathrm{H}_{\text {gas }}+\mathrm{H}_{\mathrm{ad}} * \text { graphite --------> } \mathrm{H}_{2}\left(\mathrm{v}^{\prime}, \mathrm{j}^{\prime}\right)+\text { graphite }
\end{gathered}
$$

The graphite temperature is $\mathrm{T}_{\mathrm{S}}=500 \mathrm{~K}$. We note that there is a large mass effect on the recombination dynamics and that $\mathrm{D}$ atoms recombine less effectively than hydrogen. A large effect on the energy threshold for recombination of the two isotopes is also observed.

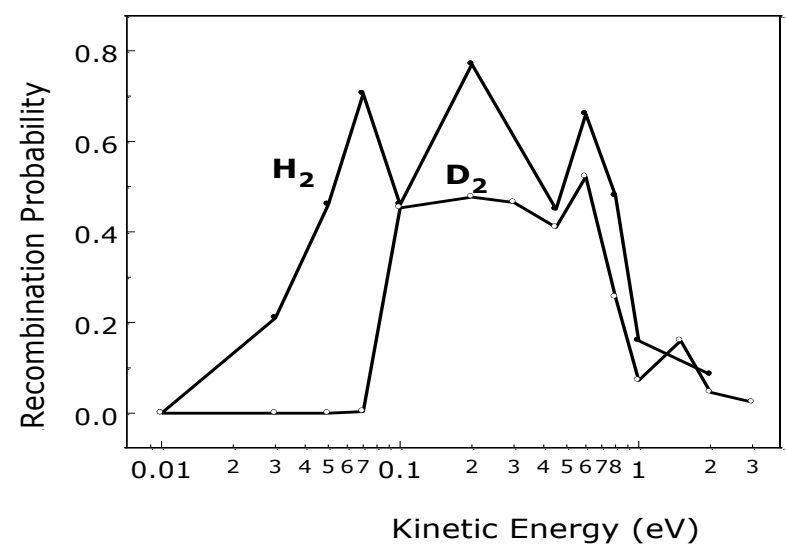

FIGURE 5. E-R recombination probabilities for $\mathrm{H}_{2}$ and $\mathrm{D}_{2}$ formation on graphite $(0001)$ at $\mathrm{Ts}=500 \mathrm{~K}$ as a function of the kinetic energy of the gas-phase isotope.

A further important factor that controls the surface catalytic behaviors is the specific surface site where the interaction occurs. This effect is relevant when the surface is corrugated, while it is not-influent for flat surfaces. In the former case, instead of a single site the surface exhibits a distribution of active surface sites each of which having different binding energy and energy barriers to reactions. This effect has been also explored in semiclassical MD calculations for $\mathrm{CO}$ formation and $\mathrm{CO}$ oxidation catalyzed by $\mathrm{Pt}(111)$ [21].

\section{REMARKS}

In this contribution we have given few examples of results that can be obtained in semiclassical molecular dynamics simulations of elementary molecular surface processes due to the interaction of O,N,H,CO with surfaces of different nature. The calculated data and the effects explored in these studies ( in particular, the energy sharing mechanism) are generally rather poorly known and not yet easily accessible in experimental observations. In this context, MD approaches can be of great importance as predictive tools in molecular surface science. Besides, the data obtained from MD calculations can be a valuable source of collisional data needed in kinetic modelling of 
various gaseous and plasma systems based on the chemistry of oxygen, nitrogen, hydrogen and used in basic and applicative research areas.

Finally, we remark that a number of other important factors that control the catalytic response of surfaces are yet to be explored in MD studies, as, for example, the influence of surface steps and corrugations and the different reactivity of amorphous substrates with respect to crystalline surfaces.

\section{REFERENCES}

1. A.K. Kurnosov, A.P.Napartovic, S. Shnyrev and M. Cacciatore, Plasma Sources Sci. Technol. 19,045015(2010)

2. M. Lino da Silva, J. Loureiro and V.Guerra, Plasma Sources Sci. Technol.18, 034023 (2009)

3. G.D. Billing and M.Cacciatore, Chem.Phys.Lett. 113, 23 (1985)

4. J. Harris and B. Kasemo, Surf.Sci. 105, L81 (1981)

5. G.D.Billing, Dynamics of Molecule Surface Interactions, NewYork: Wiley (2000)

6. M.Cacciatore, G.D.Billing, Surf. Sci. 232, 35 (1990)

7. L.Bedra, M.Rutigliano, M. Cacciatore, M. Balat-Pichelin, Langmuir 22, 7208 (2006)

8. M.Cacciatore and M.Rutigliano, Plasma Sources Sci. Technol. 18, 023002 (2009)

9. A.E.Depristo and A.Kara, Adv.Chem.Phys. 77,163 (1990)

10. M.Cacciatore, M.Rutigliano and G.D.Billing, J.Thermophys.Heat Transfer 13, 195(1999)

11. M.Rutigliano, C. Zazza, N. Sanna,A.Pieretti, G.Mancini, V.Barone and M.Cacciatore, J.Phys.Chem. 113, 15366 (2009)

12.M.Rutigliano, A.Pieretti, M.Cacciatore, N. Sanna and V.Barone, Surf.Sci. 600,4239 (2006)

13. C.Arasa, H.F.Busnengo, A.Salin and R.Sayòs, Surf.Sci. 602, 975 (2008)

14. S.C. Creighan, J.S.A.Perry,S.D.Price, J.Chem.Phys. 124,114701(2006)

15. V.Pirronello, C.Liu,J :E.Roser, G.Vidali, Astron.Astrophys. J. 344,681(1999)

16. see for example : S.Morisset, F.Aguillon, M.Sizun, V.Sidis, J.Chem.Phys. 121,6493 (2004);

R.Martinazzo, G.F.Tantardini, JChemPhys 124,124703(2006);

A.J.H.M.Meijer, A.Farebrother, D.C.Clary, A.J. Fisher, J.Phys.Chem. A105, 2173(2001)

17.E. Congiu, E. Matar, L. E. Kristensen, F. Dulieu, and J.L.Lemaire, Mon. Not. R. Astron. Soc. 3,L96(2009)

18.a) M.Rutigliano, M.Cacciatore and G.D.Billing, Chem.Phys.Lett. 340,13 (2001)

b)M.Rutigliano and M.Cacciatore, Chem.Phys.Chem. 9,171 (2008)

19. B. Kerkeni, D. C. Clary, Chem.Phys. 338,1 (2007)

20. S. Morisset, F. Aguillon, M. Sizun, and V. Sidis, J.Chem.Phys.122, 194702 (2005)

21. M. Cacciatore, E. Christoffersen, M. Rutigliano, J. Phys. Chem. A 108,8810( 2004);

G.Billing and M.Cacciatore, Chem.Phys. 103, 137(1986) 